\title{
Subject Index Vol. 5, 1994
}

p/A4 protein deposition I

Aging 110,153,266,310

Alzheimer dementia 119

Alzheimer's disease 1,6,12,36,42,69,88,99, 106,110,148,168,193,203,210,215,229, 234,240,243,247,252,266,295,302,310, 314,327,334

Amyloid 1,210

- angiopathy 210

- precursor protein 62 P-Amyloid protein 215 Antidiuretic hormone 295 Apolipoprotein E 240 Arterioles 153 Astrocytes 62 Attention 266

Basal forebrain 57 Binswanger's disease 79,148,177 Brain 69

- $\quad$ cortex 6

- imaging 193

CAMDEX 257 CANTAB 266 Capillaries 153 Case-control study 314 Cat 168

Centenarians 106, 348 Cerebral blood flow 327

- hemorrhage 210

- $\quad$ ischemia 168 Cerebrospinal fluid 295 Cerebrovascular dementia 145

- disease 137 Classification 145

Clinical and immunohistochemical studies 12

- deterioration 36 Clonidine 243 Cognition 203,266 Cognitive decline 334

- symptoms, prevalence 99

- $\quad$ test battery 266

- $\quad$ tests 289

- $\quad$ training 88 Collagen 153

Correlations, clinicopathologic 348 Cortical synaptophysin expression 79 Corticotropin releasing factor 295 Cytogenetic analysis 310 Cytoskeleton 168

Delta sleep inducing peptide 295

Dementia 12,17, 23, 29, 42, 48,106,133,137,

148,185,193,210,229,289,339 -, frontal type 240 Dexamethasone suppression test 252 Diagnostic criteria 189 Disability 289 Discriminant analysis 69 Down syndrome 69

EEG 88,119

- frequency analysis 23

Elderly 289, 302 
Endothelin-1 153 Epidemiological methods 137 Executive function 177 Extreme aging 106

Factor analysis $266{ }^{18}$ FDG 69' First admission 29 Fluorescence 234 Frontal cortex 1,36

- lobe dementia 42

Frontal-subcortical circuits 177

Gait disorders 197 General hospital patients 29

Geographical differences 110

Hereditary cerebral hemorrhage with amyloidosis (Dutch) 210

- disease 153

Hippocampus 110,229

Hospital statistics 339

Hypertension 174,197

Hypertensive vascular disease 174

Hypoperfusion 145

Immunohistochemistry 57,153 Incidence rate 29 Ischemic stroke 197

Lacunes 177

Language impairment 334

Large and small vessel disease 145

Leukoaraiosis 148

Lewy body disease 215

Lipofuscin 229,234

Longitudinal analysis 36,334

- $\quad$ concordance 99

Magnetic resonance imaging 48,174,193 Memory 266 Mental status 106

- $\quad$ - examination 17 Microscopy 234 MMSE 266,334 Morphometry 57 Motor disability 23

- $\quad$ neuron disease 42

Multi-infarct dementia 130,133,181,185 Multiple sclerosis 48 Multiubiquitin chain 282 Myelin lipids 163

Nerve growth factor receptor 57

Neuritic plaques 110

Neurochemical changes 163

Neurofibrillary tangle(s) 110,215,229,247,348

Neuropeptide Y 295

Neuropsychological evaluation 88

- $\quad$ tests 48

Neuropsychology 203

Neurotransmitters 163

Normal elderly 119

Occipital cortex 36 
Paired helical filaments 282 Parahippocampal gyrus 1 Parkinsonian dementia 17 Parkinson's disease 12,17,23,215,266 Phosphatidylserine 88

Phosphorylation 282 Physostigmine 243 PIP kinase 6 Population surveys 289 Positron emission tomography 69,88 Prevention of dementia 314 Primary progressive aphasia 334 Proline-directed protein kinase 282 Psychological testing 257 Psychosocial inactivity 314 Pyramidal neurons 62,229

Record linkage 339

Regional cerebral blood flow 302

- $\quad$ - glucose metabolism 36

Review 137

Riskfactorfs) 137,314,339

p-Secretase 62 Senile dementia 106

- $\quad$-, Alzheimer type 252

- $\quad$ plaques 247,348 Sensitivity 257

Single photon emission computed tomography

302 Sister chromatid exchanges 310 Somatostatin 295 Specificity 257

St rat egic-infarct dementia 181 Stroke 137

Subcellular localisation 6 Subcortical dementia 177

- infarction 197

- $\quad$ white matter dementia 181 Sulci and gyri 1

Tacrine 295

Tau 168,282

- $\quad$ protein 215

${ }^{99 m}$ Tc-hexamethylpropyIeneamine oxime 302 Temporoparietal cortex 36 Tetrahydroaminoacridine 295

Thalamus 36

Transcranial Doppler sonography 327

Twin studies 99

Type 1 PI kinase 6

Ubiquitin 168 Ubiquitination 282

Validation procedures 257 Vascular cognitive impairment 130

- $\quad$ dementia 79, 130, 133,137, 153, 163,174, 185,189,193,197,203,240,252

- disease 133

Vasoactive intestinal peptide 295 Visual cortex 247

- $\quad$ evoked responses 247

White matter changes 148

- $\quad$ - lesions 197 